\title{
On spurious allusions
}

\author{
EVAN HARRIS WALKER \\ U.S. Army Ballistic Research Laboratories, Aberdeen Proving Ground, Maryland 21005
}

\begin{abstract}
The criticisms of Walker' theory by MacLeod, Virsu, and Carpenter are shown to stem from a too simple reading of the text of the theory, a failure to investigate fully the references cited to support their position, and an effort to bolster the theory of lateral inhibition in orientation detectors. The criticisms are shown to misrepresent the experimental data of various reports. Walker's physiological premises are shown to be sound, and the cases of "contrary evidence" are shown in fact to be in agreement with the theory. Lateral inhibition in orientation detectors as an explanation of optical illusions is shown to fail completely to agree with experimental results.
\end{abstract}

It is perhaps the postponement of using fully the mathematical tools available to the psychophysicist that delays our understanding of such complex phenomena as optical illusions. Even quite simple systems whose workings are fully understood can manifest behavior that is unexpected if not formulated and checked mathematically. In a mathematical framework, details of the theory are subject to careful analysis serving to elucidate subtle points.

Take, for example, MacLeod, Virsu, and Carpenter's (1974) discussion of vertices of illusion figures. It is stated that a figure in which "the vertices are removed to the extent that the converging lines never come as close to each other as $\lambda$ (Fig. 2, p. 469) should show no effect whatever." The reader should first appreciate how precisely this criticism can be posed, a benefit of the prcision of the theory. The authors then reference the studies of Cumming (1968) to disprove the theory.

The Cumming's criticism of Chiang's (1968) diffraction theory, previously considered by Walker (1973), gives a variant Poggendorff figure omitting a small segment of the transversal line and states, without citing experimental evidence, that "the illusion still occurs even when the lines do not intersect." Weintraub and Krantz (1971), however, show experimentally that the removal of such small segments significantly reduces the magnitude of the Poggendorff illusion, in agreement with Walker's theory. The illusion in fact becomes, according to their data, half as large. A nonzero effect in the case of such gaps should be expected to arise from higher order tigural processing by the central nervous system (see Rosenfeld \& Lipkins, 1970) which fills obvious gaps in figure reconstruction. Spurious excitations then arise in the central nervous system for these reconstructed lines. (We return to this point below.) MacLeod, Virsu, and Carpenter, fully cognizant that their argument depends on the value of $\lambda$, have nevertheless failed to evaluate and compare this quantity with the results of precise experiments (Weintraub \& Krantz, 1971) before making their criticism, and the experimental evidence shows their contention to be false.

Since much of the poignancy of the MacLeodVirsu-Carpenter argument would come from the theory of lateral inhibition in orientation detectors (LIOD) and Walker's (1973) comments on that theory, it is necessary to show inadequacies in that theory before countering the further criticisms MacLeod, Virsu, and Carpenter raise against the present theory. The criticism of these authors given above makes clear that the LIOD theory (Blakemore, Carpenter, \& Georgeson, 1970) is not distancedependent and does not depend on vertices. As a result, that theory cannot account for the Weintraub and Krantz (1971) data for small segment removal at vertices. Further, the experiments of Tong and Weintraub (1974; see their Fig. 4 and text) are devastating to the LIOD theory. These authors study a Poggendorff variation in which the magnitude of the illusion is plotted against the position of the diagonal end point. Not only do they find that the greatest error occurs when the transversal displacement is zero (vertex fully formed), in complete agreement with Walker's theory, but the magnitude of the illusion falls rapidly if the line extends beyond the vertical, crossing that line. Tong and Weintraub conclude: "The prime requisite for the occurrence of a Poggendorff effect is the existence of an actual intersection between transversal and parallel [p. 267]." MacLeod, Virsu, and Carpenter make quite clear that this factor is entirely lacking in the LIOD theory. We are forced to conclude that the LIOD theory is not valid.

It is also appropriate at this point to examine the research note of Blakemore and Tobin (1972) 
referenced as physiological substantiation of the LIOD theory of optical illusions. Basically, the experiment shows that for an orientation detecting receptive field, the response of that field is reduced if the line exciting that field is part of a background of parallel lines. In this, the response of orientation detectors does not differ from lateral inhibition in uniform retinal illumination. But does this inhibition generate the response required by Blakemore, Carpenter, and Georgeson (1970)? In that paper, they present (their Fig. 1) the response or "activity that might be set up in the orientation domain" by individual lines and pairs of lines of differing orientation. The theory demands a special response of the receptive field for individual lines as a function of orientation. One must have as a function of the orientation angle (i) a broad peak, about $0 \mathrm{deg}$, (ii) a steep slope into the inhibitory region, and (iii) a sudden, fairly rapid rise as the orientation angle increases further. The experimental work (Blakemore \& Tobin, 1972) indicates a sharp peak that falls in intensity off orientation, but does not show the required inhibition for single lines off axis.

As the Blakemore and Tobin data stand, they do not suffice to establish the requirements of the LIOD theory of optical illusions, though the data do show the existence of lateral inhibition in orientation detectors, which has its own significance. Since this experiment was conducted largely to test the lateral inhibition theory of optical illusions, one might wonder why a simple test of lateral inhibition using only two lines was not undertaken. This would have been more specific to the requirements of the prior paper by Blakemore, Carpenter, and Georgeson. We must conclude that the LIOD theory is a hypothesis, unconfirmed by physiological data and contradicted by psychological measurements.

Houssiadas and Brown (1963) showed that in certain figural illusions involving intersecting lines, specifically the Zöllner, Hering, and Wundt figures, the illusion disappears at large viewing distances. In the fovea centralis, extended receptive fields do not occur, so that for images falling principally on the fovea centralis one should expect from Walker's theory that the illusion, while not totally absent (as misrepresented by MacLeod, Virsu, and Carpenter), should be significantly reduced (the predicted magnitude should properly be calculated for specific cases). For some reason, MacLeod, Virsu, and Carpenter point out this fact as though it would indicate a shortcoming of theory; in fact, from Houssiadas and Brown, this is further support for the theory.

MacLeod, Virsu, and Carpenter state, "A second vital assumption ... is that excitation can and does occur between two lines separated by less than five receptive tield center diameters, but does not invade the region outside the pair of lines [Walker, 1973.
Fig. 2 and p. 470]." Figure 2, of course, is merely schematic, and the term between is used only in the elementary formulation, whereas integration over the entire space of the figure is carried out in the more detailed formulation specified in Appendix A of that paper. Actually, receptive fields outside pairs of lines will contribute and have been included in the elementary calculation as contributing to the number of line-clement excilations, $N$, which is the correct treatment. Nothing has been improperly formulated.

MacLeod, Virsu, and Carpenter, in discussing the treatment by Walker of the receptive field center and surround, have ignored a significant point. Walker (1973) states, "It is worth noting, however, that Enroth-Cugell and Pinto (1972) have shown that the pure surround response to off-center receptive fields is similar in shape to the pure center response of on-center cells." Walker (1973) also states, in discussing the Weintraub-Krantz paper, "This line is established not with respect to an absolute perception space but with respect to the immediate data field...." Now if the off-center pure surround response is substantially the same as the on-center pure center response, how does the brain distinguish the two? The present theory assumes (and this assumption is subject to experimental verification) that for line detection it does not. There is not an absolute perception space but a data field that must be structured through cortical data processing. Lines crossing the surround of an off-center receptive field will evoke responses like lines crossing the center of an on-center field. Thus, in the MacLeod-VirsuCarpenter criticism, whereas these authors recognize that "center and surround responses are of opposite polarity," they fail to recognize that there exist both on-center and off-center fields, responding as mirror processes of each other.

MacLeod. Virsu, and Carpenter, in their issue over the data of Enroth-Cugell and Pinto (1972), have correctly noted that the latter showed the pure center and pure surround represent "algebraic summation," but have failed to understand that this summation is incorporated into Walker's Eq. 61; they have failed furthermore to recognize the essential distinctions in the way Enroth-Cugell and Pinto explain their experimental data and the theoretical treatment given by Walker. Enroth-Cugell and Pinto treat the receptive field as consisting of two sectors, the pure central and pure surround. This is an artifact, useful for the discussion of their data but not reflecting the actual physiology responsible for those data. The responses of these two sectors are shown to be algebraically summed to obtain the ganglion cell response for the receptive field. The treatment by Walker models the receptive field in terms of its individual receptors, which transmit their excitation to the ganglion cell. The response measured at the ganglion cell is taken to be the albegraic sum taken 
over the tield of receptors; that is to say, an integral over the alrea (in the limit of a substantial number of receptors in the field). We could symbolically have divided the integral (Walker's Eq. 61) into two parts.

$$
\begin{aligned}
\mathrm{S} & =\int_{\text {surface }} \mathrm{Eds}=\int_{0}^{2 \pi} \int_{0}^{\rho_{0}} \operatorname{E} \rho \mathrm{d} \rho \mathrm{d} \theta+\int_{0}^{2 \pi} \int_{\rho_{\mathrm{o}}}^{\rho_{1}} \operatorname{E} \rho \mathrm{d} \rho \mathrm{d} \theta \\
& =\mathrm{S}_{\mathrm{c}}+\mathrm{S}_{\mathrm{s}},
\end{aligned}
$$

where $S$ is the magnitude of the excitation signal received by the ganglion cell and $E$ is the excitation, defined in Walker's Eq. 58, transmitted by receptors in a given element of area $\mathrm{dS}=\operatorname{\varrho d} \varrho \mathrm{d} \Theta$, with the region $0 \leqslant \varrho \leqslant \varrho_{0}$ defining the central and $\varrho_{0}<\varrho \leqslant \varrho_{1}$ defining the surround of the receptive field. We see that the pure central excitation, $S_{\mathrm{c}}$, and the pure surround, $S_{S}$, sum to give $S$. The statement by Walker that the effect of the surround (on the response of a receptor will be multiplicative arises from an appreciation for the distinction between experimental procedure to elicit a pure surround response, for example, and the treatment required to model the receptor response. A pure surround response required that the central region of the receptive field be illuminated. Without that illumination, the surround cihibited a weak response, in keeping with Walker's equations. Thus, this adaptive illumination serves to molulate the response of the neighboring receptors, heading to the $F_{1} F_{2}$ term incorporated in Eq. 61 . Walker's statement is thus seen to be fully in accord with the experimental data both as regards multiplicative effects and the statement by Enroth-Cugell and Pinto regarding algebraic summation manifestations in their data. The criticism by MacLeod, Virsu, and Carpenter is thus the result of their failure to grasp the meaning of the mathematics.

It should be appreciated that the treatment given by Walker does not require the introduction of separate types of receptors to model the central and surround regions, as would be implied by Enroth-Cugell and Pinto. However, it should be understood that a more complete treatment is called for to meet the requirements of all of the data given by Enroth-Cugell and Pinto in order that the time dependence they give be accounted for. This should involve both a decay in excitation as a function of time and effects due to the retarded excitation [accounting for the time delay in propagation of the excitation by use of a factor $\xi-a t$ (where $a$ is the reciprocal propagation velocity) for $\xi$ in Walker's Eq. 60].

We have shown above that the principal objections presented by MacLeod, Virsu, and Carpenter are not properly founded and arise from shortcomings in their appreciation of the mathematical theory of figural illusions presented by Walker. Furthermore, we have shown that the LIOD theory, far from being established, clearly lacks physiological justification and lails to satisfy specific experimental data, notably that of long and Weintraub. We now turn to an important point ratised by MacLeod, Virsu, and Carpenter concerning the difference between the data of Blakemore et al (1970), involving as it does only acute or obtuse angles, and the treatment by Walker of figures which generally contain both acute angles and the complementary obtuse angles.

Resolution of this point is simple but involves higher order processes in the figural analysis of the central nervous system than those already introduced into the theory. Since additional processes demand additional adjustable coefficients, this point was specifically omitted by Walker (1973) to retain a strictly first-order theory with one coefficient. As mentioned above, the data of Weintraub and Krantz (1971) show that the omission of line segments near intersections of lines changes the magnitude of the illusion by a factor of 0.5 (from $22.70 \mathrm{~mm}$ in the unaltered Poggendorff figure to $11.00 \mathrm{~mm}$ for the interrupted vertices). It was also stated above that figural processing in the central nervous system should (see Rosenfeld \& Lipkin, 1970) reconstruct line elements of figures, and data processing on such reconstructed figures would also lead to spurious excitations centrally. While such processes have not been established physiologically, their effect can be calculated. In particular, in the case of the Blakemore et al (1970) experiment, for obtuse angles, the comparison line adjusted by the Ss extends to a point close to the displayed obtuse angle. Assuming a coefficient $\beta=0.5$, one can calculate the effect for obtuse angles. The result is shown in Fig. 1 together with a seventh-order polynomial least squares fit to the Blakemore et al (1970) data. The fit obtained is good. This added concept should be of value in treating illusions such as produced by the Ponzo figure and illusions involving higher central nervous system processing.

MacLeod, Virsu, and Carpenter raise the question of the significance of experiments involving stereoscopically formed figural illusions for the theory presented by Walker (1973); they cite in this regard the work of Julesz (1971), who has used random-dot stereograms to study figural illusions. MacLeod, Virsu, and Carpenter feel it to be significant that Walker "omits to mention" the work of Julesz since "in cyclopean view, processes in the monocular pathways are unable to contribute to the illusion, yet the Poggendorff and Müller-Lyer illusions appear to their full extent when observed in this way." There was, however, no intent to avoid this subject. While Julesz (1971) and the papers by Papert (1961) and Hochberg (1963), which he cites, were not previously referenced, eight papers on the general controversy concerning stereoscopic observation of geometric 


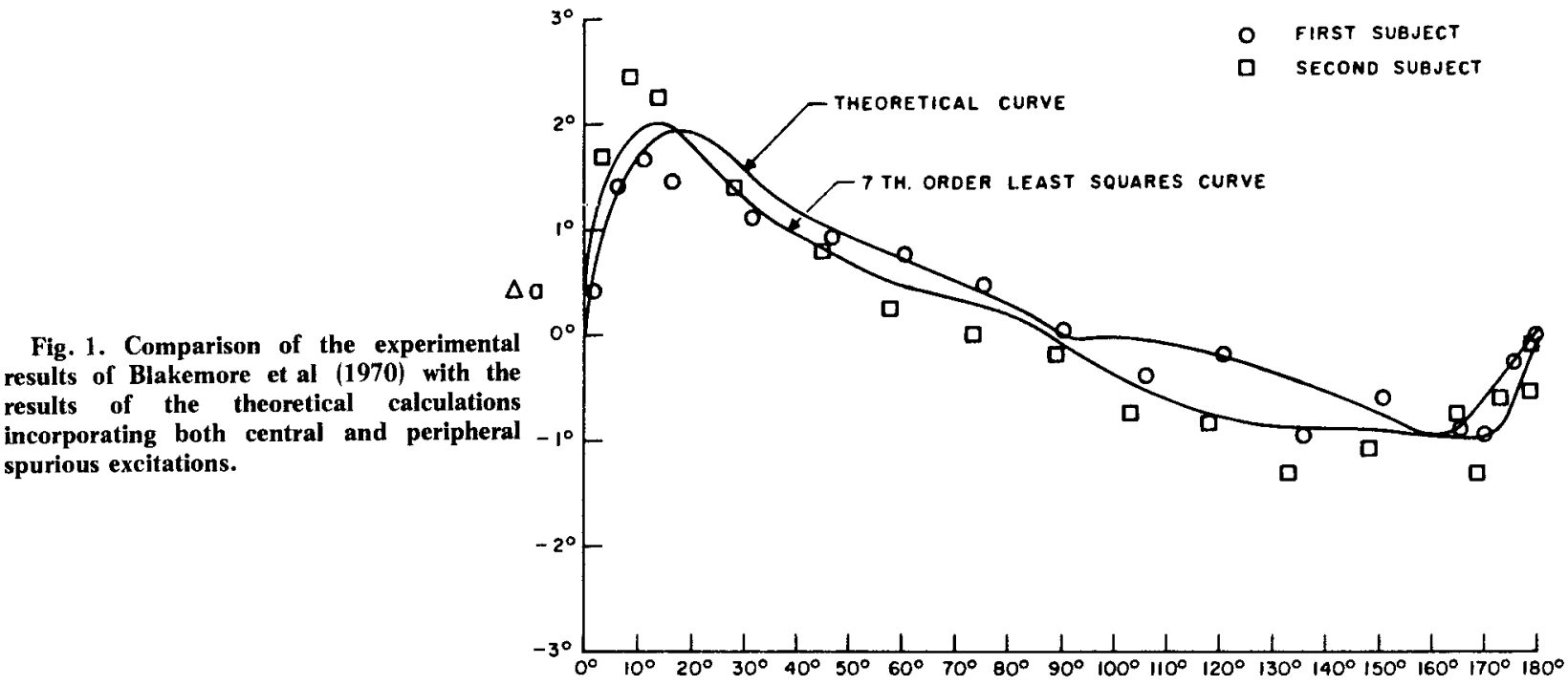

illusions were referenced: these were the papers by Ohwaki (1960). Springbett (1961), Witasek (1899). Lau (1925), and Shiller and Wiener (1968), as well as the works by Day (1961), Boring (1961), and Gregory (1968), who were not referenced by Julesz.

The questions raised by the work of Julesz (1971) in which random dot stereograms have been used to. study optical illusions are far more involved than one would be led to believe from the comments of MacLeod, Virsu, and Carpenter or for that matter by Julesz. First, it should be noted that there is a controversy concerning the proper interpretation of the data reported by these various authors, so it is with trepidations that this author proceeds below to relate these experiments to the theory. However, it is the mark of a good theory that it can frequently shed light on the cause of apparently conflicting experimental results.

It has been generally maintained, since Witasek (1899), that if the classical phenomenon is the same, both qualitatively and quantitatively, as the stereoscopic or "cyclopean" phenomenon, then we can conclude the illusion to be caused by central processing. We will see below that, in fact, this is not necessarily the case. It is possible for both central and peripheral processing to contribute to both line element and spurious excitations in such a way that the removal of the peripheral contributors to the perceived image reduces line element and spurious contributions proportionately leaving the relative magnitudes of centrally produced line-element excitations and spurious excitations unchanged. Nevertheless, considerable effort has been made to obtain data of this type to determine if stereoscopically produced illusions are the same or different from the classical illusions. Two principal techniques have been employed: (1) stereoscopic formation of the illusion image in which the inducing elements of a variety of geometric illusions are presented to one eye and the test elements to the other, and (2) stereoscopic formation of the illusion image using a correlated pair of random-dot patterns (random to monocular vision but containing an apparent three-dimensional image incorporating the entire illusion when viewed stereoscopically). These will be referred to below as the "split figure" and "random dot" stereoscopic techniques, respectively.

Julesz (1971) has constructed random-dot stereograms of the Poggendorff and Mueller-Lyer illusions, among others, but does not report having conducted quantitative experiments using these figures. Julesz, citing the work of Papert (1961) and Hochberg (1963), states that the result indicates "not only a qualitatively but a quantitatively exact agreement [p. 31]" using this technique. However, Papert (1961), reporting on the Mueller-Lyer illusion, does not give any quantitative data, does not state the number of Ss used in the test, and does not state that the results are quantitatively in agreement with the classical illusions. The work of Hochberg (1963) may be quantitative, but a reference to published results is not given. In a footnote, Julesz references the works of Ohwaki (1960), Springbett (1961), Witasek (1899), and Lau (1922) on split-figure stereoscopic experiments each reporting reductions or absence of geometrical illusions under stereoscopic observation, together with the data obtained. Julesz summarily states: "The correct interpretation of these results is that the reason for the diminished illusions is binocular rivalry." Julesz does not, however, cite the work of Day (1961), which is the source for a detailed study of this point.

In split-figure stereoscopic observation, binocular rivalry is reported. Day conducted experiments of this 
type using 10 psychology graduate students as Ss. The use of such $S s$ was most valuable, since these Ss gave quite detailed reports of their observations. The detail of the reports may, however, exaggerate the importance of rivalry, since, despite the general occurrence of binocular rivalry, most of both images can generally be seen simultaneously in such stercoscopic tests and at times both images are fully observed together with a report of a reduction in the magnitude of the illusion. It is not appropriate to detail here Day's arguments; however, it cannot be said that he concludes flatly that rivalry is fully adequate to explain the reduced magnitude of the illusion whereas Ohwaki and Springbett conclude that rivalry does not provide an explanation of the results. Day concludes: "Even if the conclusions reached by Ohwaki and by Springbett were acceptable, their 'explanation' of the origin of illusion li.e., as being peripheral] fails to explain the presence of reduced illusory effects in stereoscopic presentation." In this criticism, Day is certainly correct; we will see below that the present theory does, in fact, account quantitatively for the reduced effect. It appears to this reader that this correct judgment of Ohwaki's and Springbett's works has caused Day to overstress rivalry as an explanation. The data given by all Es using the "split-figure" test shows the presence of a reduced illusion when both images are fully observed, and this is in accord with the present theory as discussed below. ${ }^{1}$ We are taking the position here that spurious excitations arise both peripherally and centrally, as detailed in the original paper (Walker, 1973) and as expanded upon above. It is felt that the data of Witasek, Lau, Ohwaki, Springbett, Day, Gregory, Schiller, and Wiener are compatible with the existence of both peripherally and centrally produced spurious excitations as well as excitations that arise because of the limited resolution of the field of neural excitations that are the brain's representation of the figural elements. How this occurs is discussed further below.

Let us now see how the random-dot stereoscopic results are to be understood within the theoretical framework. MacLeod, Virsu, and Carpenter, and Julesz, as well, appear to assume that if peripheral processes are causally related to optical illusions, those characteristics of the peripheral processes that enter into the formation of the visual illusion will be eliminated using the random-dot approach. This is not the case, however. Exactly the same type of excitations still occur in all regions of each monocular view simply because each view consists of neighboring dots. Of course, monocularly, these excitations, which are not spurious relative to the monocular views, are uncorrelated with the elements of the geometric illusion figure. But in the case of the random-dot tigures, a new central process enters to correlate and "sort" excitations from each eye. Which excitations should be ascribed to the background and which to the geometric figure is subject to ambiguity due to the finite resolution of the neural excitations that represent the image. The effort to represent a continuous figure in terms of a finite array of excitations (data points), by whatever algorithm, must lead to some systematic errors. In the present case, excitations arising at points near both the inducing and test images would have a higher probability of being ascribed as a part of the geometric figure than of the background. The magnitude of this effect is determined by the resolution of the neural net on which the image is represented, just as for the classical figure. Thus, we should expect the illusion to be of the same magnitude.

There is an alternative way of stating this. As we have seen above, we assume that spurious excitations arise not only peripherally, but also centrally. In the classical case, contributions to the line element excitations occur both peripherally and centrally as the brain processes the data that represent the figure. The magnitude of the illusion is determined by the ratio of the spurious to line-element excitations, as detailed by Walker' theory. In the case of random-dot stereograms, neither peripheral line-element excitations nor spurious excitations correlated to line elements arise peripherally. Correlated spurious excitations and line-element excitations both arise centrally. The ratio of spurious to line-element excitations forming the perception of the figure is the same as classically and the magnitude of the resulting illusion should be the same in keeping with the qualitative reports of Papert (1961), Hochberg (1963), and Julesz (1971).

In the random-dot stereograms, the test and inducing figures can occur at different apparent depths. When this is done for the Poggendorff figure, the illusion " is greatly reduced or even disappears [Julesz, 1971]." To achieve a difference in the perceived depth of the inducing and test elements of the figure, the two monocular images must differ even for those areas that form part of the geometric illusion. In the case of the Poggendorff, this difference occurs at the intersection between the diagonal line and the vertical bar (referring to the Julesz figure). The two views differ in such a way that the transverse will be seen by one eye extending "under" the edge of the bar as viewed by the other eye. That is to say, the transverse extends beyond the edge of the vertical bar. This is the same situation in which Tong and Weintraub (1974) showed that the magnitude of the illusion falls rapidly if the transverse line extends beyond the vertical line (the edge of the bar) so as to cross that line, in agreement with the theory. Thus, in the case of random-dot stereograms showing the test and inducing figures at differing aparent depth, the 
The Effect of Binocular Viewing Conditions on the Occurrence and Magnitude of Optical lllusions for the Poggendorff Figure

\begin{tabular}{|c|c|c|c|c|c|c|}
\hline & \multicolumn{2}{|c|}{$\begin{array}{c}\text { Peripheral } \\
\text { Contribution }\end{array}$} & \multicolumn{2}{|c|}{$\begin{array}{c}\text { Central } \\
\text { Contribution } \\
\end{array}$} & \multirow{2}{*}{$\begin{array}{c}\text { Ratio: } \\
\text { Spurious/ } \\
\text { Line }\end{array}$} & \multirow[b]{2}{*}{$\begin{array}{l}\text { Resulting } \\
\text { Illusion }\end{array}$} \\
\hline & $\begin{array}{c}\text { Line } \\
\text { Elemen }\end{array}$ & $\begin{array}{l}\text { Spuri- } \\
\text { it ous }\end{array}$ & $\begin{array}{c}\text { Line } \\
\text { Element }\end{array}$ & $\begin{array}{l}\text { Spuri- } \\
t \text { ous }\end{array}$ & & \\
\hline Classical observation & $\mathbf{N}$ & $\mathrm{n}$ & $\mathbf{N}$ & $\mathrm{n}$ & $\mathrm{n} / \mathrm{N}$ & Full \\
\hline $\begin{array}{l}\text { Stereoscopic observation of inducing and test figure } \\
\text { (split-figure test) }\end{array}$ & $\mathbf{N}$ & 0 & $\mathrm{~N}$ & n & $\mathrm{n} / 2 \mathrm{~N}$ & $\begin{array}{l}\text { Magnitude } \\
\text { reduced }\end{array}$ \\
\hline Random-dot stere oscopic observation, standard & $0 *$ & $0^{*}$ & $\mathrm{~N}$ & $\mathrm{n}$ & $\mathrm{n} / \mathrm{N}$ & Full \\
\hline Random-dot stereoscopic, inducing and test figures separated & $0^{*}$ & $0^{*}$ & $\mathbf{N}$ & 0 & 0 & No illusion \\
\hline
\end{tabular}

*Excitations present peripherally but not correlated to elements of figure.

present theory indicates that the illusion would be absent. The stereoscopic observations are thus fully in accord with the present theory. The differences encountered in the classical observation, split-figure test, random-dot test with inducing and test figures in the same plane, and random-dot test with stereoscopically separated planes is shown in Table 1 , where $\mathrm{n}$ and $\mathrm{N}$ refer, respectively, to the number of spurious and line-element excitations in each case.

From our previous discussion of the contribution of higher order processes in the figural analysis of the central nervous system, as used to obtain Fig. 1, and from the relative values given in Table 1 , we see that stereoscopically formed Poggendorff figures should produce a displacement half as great as that for normal viewing. Gregory (1968) reports the Poggendorff illusion to be absent in 29 of 42 cases tested stereoscopically, and Day (1961) reports it absent in 4 of 10 cases (average ratio for the two experiments, 0.55 ; ratio for total $\mathrm{Ss}, 0.64)$. These data, taken as a measure of the relative magnitude of the stereoscopic and normal illusion, would be in complete agreement with the coefficient 0.5 used above and in the calculation of Fig. 1. Unfortunately, these experiments do not satisfactorily quantify the magnitude of the illusion; further testing of this point is called for. Nevertheless, we see that the objection raised by MacLeod, Virsu, and Carpenter is based on an incomplete consideration of the relevant reference material as well as a superficial consideration of the theory. Rather than being inadequate to account for Julesz's results, the theory allows us to understand the causes of conflicting experimental results.

MacLeod, Virsu, and Carpenter also raise the question of the orientation effect on the Poggendorff illusion; they state that "the orientation effect is easily explained-indeed expected-on the basis of cortical interactions between orientation detectors, but is incompatible with Walker's model." These proponents of the LIOD theory have not produced physiological evidence to support this claim, nor have they derived such results. They merely mean that if one makes the several assumptions, not only regarding the nature and magnitude of these interactions, but, in addition, regarding their functional character, one may also assume that the various coefticients in their theory will also be functions of the angles. Such functional dependence of the coefticients could serve equally in either theory. Being more constructive on this point, however, we can state that since the orientation receptive fields, as orientation detectors for line elements, approximate to the least squares procedure as a limit process, a greater acuity in this process for vertical and horizontal lines would largely account for the observed effects. The transverse line in the vertical position would, as a result of this acuity, serve as the reference line and thus reduce the illusion as computed by Walker's theory.

Finally, MacLeod, Virsu, and Carpenter admit to being unable to understand a most elementary point concerning figural illusions: the cortical perception of figures differs from that displayed on the retina. In discussing Walker's treatment of the experiments of Weintraub and Krantz (1971), these authors state: "We cannot understand how spurious excitations generated by the sides of an acute angle could displace the apparent apex..." But, if the apex cannot appear where it is not, how then can the line? The basic lession to be learned from figural illusions is that, since figures are not perceived as displayed, the retinal image is not transferred as such to the brain, but must be reconstructed by the brain's data processing. It is for this reason that damage to the retina or distorted image projection onto the retina can generally be compensated for cortically, given sufficient time for adaptation. The cause for the displacement of the apex is discussed in the text. Referring to Walker's (1973) Fig. 8, the position of Line $A$ is not fully specified by the data available from the retinal excitations. The center line between $A$ and $B$ is not available as a reference. Only the excitations from the area between Lines $A$ and $C$, the dot on Line $\mathrm{C}$, and the spurious excitations above the true Line A caused by B serve as cues as to the orientation of Line A. The position of B is entirely established with reference to $A$. In general, this problem calls for the calculation of several quantities to determine the 
position of the perceived lines, $A$ and $B$, but the approach of the theory is straightforward.

The author would like to acknowledge (in response to private communication) that "neural blurring" was mentioned by L. Glass (1970) in his treatment of optical blurring after Chiang (1968) as a possible contributor to visual illusions.

\section{REFERENCES}

Blakemore, C., Carpenter, R. H. S., \& Georgeson, M. S. Lateral inhibition between orientation detectors in the human visual system. Nature, 1970, 228, 37-39.

Blakemore, C. \& Tobin, E. A. Research note: Lateral inhibition between orientation detectors in the cat's visual cortex. Experimental Brain Resecurch, 1972, 15, 439-440.

Boring. E. G. Letter to the editors. Scientific American, 1961, 204, 18-19.

Chiang, C. A new theory to explain geometrical illusions produced by crossing lines. Perception \& Psychophysics, 1968 , 3. $174-176$.

Cumming, G. D. A criticism of the diffraction theory of some geometrical illusions. Perception \& Psychophysics, 1968, 4, 375-376.

DAY, R. H. On the stereoscopic observation of geometric illusions. Perception \& Motor Skills, 1961, 13, 247-258.

Enroth-Cugell, C., \& Pinto, L. H. Properties of the surround response mechanism of eat retinal ganglion cells and centersurround interaction. Journal of Physiology, 1972, 220, 403-439.

GLASS, L. Effect of blurring on perception of a simple geometric pattern. Nature. 1970, 228, 1341-1342.

Gregory, A. H. Visual illusions: Peripheral or central? Nature, 1968, 220, 827-828.

HOCHBERG. J. Illusions and figural reversals without lines. Paper given at the Fourth Annual Meeting of the Psychonomic Society, Bryn Mawr. Pennsylvania. 1963.

Houssiadas, L., \& Brown, L. B. The effect of viewing slant and distance on some visual illusions. Australian Journal of Psychology, 1963, 15, 108-112.

Jut.esz, B. Foundations of cyclopean perception. Chicago: Chicago University Press, 1971.

LaU. E. Versuche über das stereoskopische Sehen. Psychologische Forschung, 1922, 2, 1-4.

LaU, E. Über das stereoskopische Sehen. Psychologische Forschung, 1925, 6. 121-126.

Macleod, D. I. A., Virsu, V., \& Carpenter, R. H. S.
On mathematical illusion. Perception \& Psychophysics, 1974. 16. $417-418$

OHwaki, S. On the destruction of geometrical illusions in stereoscopic observations. Tohoku Psychological Folia, 1960, 29. 24-36.

PAPERT, S. Centrally produced geometrical illusions. Nature, 1961 , 191.733.

Rosenfeld, A.. \& Lipkin, B. S. Texture synthesis. In A. Rosenfeld and B. S. Lipkin (Ed.), Picture processing and psychopictorics. New York: Academic Press, 1970.

SHiller, P., \& Wiener, M. Binocular and stereoscopic viewing of geometric illusions. In R. N. Haber (Ed.), Contemporary theory, and research in visual perception. New York: Holt, Rinehart \& Winston, 1968.

SPRingbetT, B. M. Some stereoscopic phenomena and their implications. British Journal of Psychology, 1961, 52, 105-109.

Tong, L., \& Weintraub, D. M. Contour displacements and tracking errors. Probing 'twixt Poggendorff parallels. Perception \& Psychophysics, 1974, 15, 258-268.

WALKER, E. H. A mathematical theory of optical illusions and figural aftereffects. Perception \& Psychophysics, 1973, 13, 467-486.

Weintraub, D. J., \& Krantz, D. H. The Poggendorff illusion: Amputations, rotations, and other perturbations. Perception \& Psychophysics, 1971, 10, 257-264.

WitaSeK, S. Über die Natur der geometrischoptischen Taeunschugen. Zeitschrift für Psychologie und Physiologie der Sinnesorgane, 1899, 19,81-174.

\section{NOTE}

1. There are further data in Day's report that support the present theory by showing that binocular rivalry affects principally those parts of the optical illusion figure that are the source of spuriously ascribed excitations. Day reports (paraphrasing his Ss): (1) Orbison illusion: "The most marked rivalry occurred at the point of intersection of the sides of the square with the circles." (2) Variant of Stanford figure: "The most marked effect was that of complete suppression from time to time of the radiating lines in and immediately around the test square." (3) Poggendorff illusion: "By far the most common report was that of suppression of that part of the rectangle in the vicinity of its points of contact with the oblique test lines." These reports show the significance of the intersections in causing these illusions in that their removal through binocular rivalry causes a reduction of the illusion.

(Received for publication April 19, 1974; revision received July 23,1974 .) 